\title{
Kataraktchirurgie und Augeninnendruck
}

\author{
Diana Melancia $^{c}$ Luis Abegão Pinto ${ }^{\mathrm{a}-\mathrm{c}}$ Carlos Marques-Neves ${ }^{\mathrm{b}, \mathrm{c}}$ \\ Departments of \\ a Pharmacology and Neurosciences and \\ bOphthalmology, \\ ' Faculty of Medicine, Lisbon University,Lisbon, Portugal
}

\section{Schlüsselwörter}

Kataraktchirurgie · Glaukom · Intraokulardruck ·

Phakoemulsifikation · Trabekulektomie · Kombinierte Chirurgie

\section{Zusammenfassung}

Die Kataraktoperation ist eine der am häufigsten durchgeführten Operationen in den Industrieländern. Neben ihrer signifikanten Auswirkung auf die Sehschärfe wird der Phakoemulsifikation auch eine potenzielle Intraokulardruck(IOD)-senkende Wirkung zugeschrieben. Während die aktuelle Evidenzlage auf eine insgesamt signifikante und anhaltende IOD-Senkung nach Kataraktoperationen hindeutet, ist noch unklar, welche spezifischen okulären Merkmale dazu beitragen könnten vorherzusagen, welche Patienten wahrscheinlich von diesem IOD-senkenden Effekt profitieren würden. Dies bei Glaukompatienten klar zu benennen ist jedoch

wichtig, wenn die Kataraktoperation einen Platz in der Behandlung des Glaukoms erhalten soll. Die vorliegende Arbeit gibt einen Überblick über die Literatur zu diesem Thema und geht darauf ein, welche Mechanismen der IOD-Senkung zugrunde liegen und welche Patientengruppen mit höherer Wahrscheinlichkeit im Hinblick auf die IOD-Senkung von diesem Eingriff profitieren könnten, um so letztlich zur Optimierung der Versorgung der steigenden Zahl der Patienten beizutragen, bei denen gleichzeitig Glaukom und Katarakt vorliegen.

(c) 2017 S. Karger GmbH, Freiburg

\section{Einleitung}

Katarakt und Glaukom gelten als führende Ursachen für Erblindung weltweit (51 bzw. 8\%) [1]. In Industrienationen ist das Glaukom die zweithäufigste Ursache für irreversible Erblindung (nach der diabetischen Retinopathie); die Krankheitslast steigt tendenziell mit der Alterung der Bevölkerung [2]. Ein ähnlicher Trend ist auch bei der Katarakt zu beobachten, deren Prävalenz ebenfalls altersabhängig ist - die globale Prävalenz von 15,5\% steigt bei Patienten über 75 Jahre auf $45,9 \%$ und wird sich bis 2020 voraussichtlich verdoppeln [3]. Entsprechend sind ältere Personen auch häufig von beiden Pathologien zugleich betroffen, ein Zustand, der wahrscheinlich weiter zunehmen wird.

Vor diesem Hintergrund entwickelt sich die Kataraktoperation zu einer der häufigsten Operationen weltweit, und es liegen Hinweise darauf vor, dass der Eingriff im Hinblick auf beide Krankheiten von Nutzen ist. Die Kataraktoperation dient nicht nur der
Entfernung der getrübten Linse, sondern führt Beobachtungen zufolge auch zur Senkung des Intraokulardrucks (IOD) in Augen sowohl mit als auch ohne Glaukom, wobei dieser Effekt in unterschiedlichem Ausmaß auftritt und von verschiedenen Faktoren beeinflusst wird, darunter die Anatomie der Vorderkammer und die Winkelkonfiguration (Offen- (OWG) vs. Engwinkelglaukom (EWG)) [4]. Außerdem kann der Eingriff die diagnostische Güte gängiger funktioneller und struktureller Analysen zur Beurteilung des Glaukoms und seiner Progression verbessern, da eine visuell signifikante Katarakt hier eine Störgröße darstellen kann. Somit erscheint die Annahme sinnvoll, dass ein gemeinsames therapeutisches Vorgehen etabliert werden könnte, das die Kataraktchirurgie als Bestandteil der Glaukom-Behandlungsalgorithmen umfasst. Offen ist dabei jedoch noch, wie sich dies am besten in die klinische Patientenversorgung einfügen ließe.

Die vorliegende Übersichtsarbeit geht der Frage nach, wie die Kataraktchirurgie den IOD sowohl bei ansonsten gesunden Per-

\section{KARGER}

Fax +497614520714

information@karger.com

www.karger.com
() 2017 S. Karger GmbH, Freiburg

Accessible online at:

www.karger.com/kop
Carlos Marques-Neves, MD, PhD

Department of Ophthalmology

Faculty of Medicine, Lisbon University

Av. Prof. Egas Moniz, 1649-035 Lisbon, Portugal

bombordo.seven@gmail.com 
Tab. 1. Klinische Studien zum Effekt der Linsenextraktion auf den IOD bei Patienten ohne Glaukom, mit okulärer Hypertension, OWG, chronischem und akutem EWG

\begin{tabular}{|c|c|c|c|c|c|}
\hline Autoren & $n$ & Nachbeobachtung & IOD prä $\rightarrow$ post, mm Hg & $\triangle \mathrm{IOD}$ & $\mathrm{p}$-Wert \\
\hline \multicolumn{6}{|l|}{ Ohne Glaukom } \\
\hline Pohjalainen [6], 2001 & 137 & 25 Monate & $16,3 \rightarrow 12,7$ & $-3,4$ & 0,001 \\
\hline Shingleton [7], 2006 & 59 & 60 Monate & $15,9 \rightarrow 13,4$ & $-1,5$ & $<0,0001$ \\
\hline \multicolumn{6}{|l|}{ Okuläre Hypertension } \\
\hline Mansberger [5], 2012 & 63 & 38 Monate & $23,9 \rightarrow 19,8$ & $-4,1$ & $<0,001$ \\
\hline \multicolumn{6}{|l|}{ POWG } \\
\hline Kim [14], 1999 & 31 & 16 Monate & $18,1 \rightarrow 15,2$ & $-2,9$ & $<0,001$ \\
\hline Shingleton [15], 1999 & 61 & 12 Monate & $17,0 \rightarrow 15,9$ & $-1,1$ & n.s. \\
\hline Pohjalainen [16], 2001 & 38 & 26 Monate & $18,4 \rightarrow 15,1$ & $-3,3$ & 0,001 \\
\hline Hayashi [10], 2001 & 68 & 24 Monate & $20,7 \rightarrow 15,2$ & $-5,3$ & $<0,0001$ \\
\hline Lai [11], 2004 & 34 & 20 Monate & $20,4 \rightarrow 15,7$ & $-4,7$ & $<0,05$ \\
\hline Mathalone [17], 2005 & 24 & 24 Monate & $17,0 \rightarrow 15,1$ & $-1,9$ & n. s. \\
\hline Shingleton [7], 2006 & 55 & 60 Monate & $18,4 \rightarrow 16,6$ & $-1,8$ & 0,005 \\
\hline Poley [18], 2009 & 124 & 10 Jahre & $17,8 \rightarrow 15,1$ & $-2,7$ & $<0,001$ \\
\hline \multicolumn{6}{|l|}{ CEWG } \\
\hline Hayashi [10], 2001 & 68 & 24 Monate & $21,0 \rightarrow 15,0$ & $-6,0$ & $<0,001$ \\
\hline Lai [11], 2004 & 31 & 20 Monate & $27,4 \rightarrow 14,8$ & $-12,7$ & $<0,05$ \\
\hline Liu [12], 2006 & 29 & 3 Monate & k.D. $(>20,0) \rightarrow 14,3$ & k.D. & k.D. \\
\hline Lai [13], 2006 & 21 & 21 Monate & $19,7 \rightarrow 15,5$ & $-4,2$ & 0,02 \\
\hline \multicolumn{6}{|l|}{ Akutes EWG } \\
\hline Jacobi [8], 2002 & 43 & 24 Monate & $40,5 \rightarrow 17,80$ & $-22,70$ & k.D. \\
\hline Su [9], 2011 & 16 & 3 Monate & $48,81 \rightarrow 10,70$ & $-38,09$ & $<0,001$ \\
\hline
\end{tabular}

sonen als auch bei Glaukompatienten mit Katarakt beeinflussen könnte. Innerhalb dieses Settings liegt der besondere Schwerpunkt auf intrinsischen anatomischen und physiologischen Faktoren des Glaukoms, die den IOD beeinflussen und potenziell prädiktiv für den möglichen Effekt einer Lentektomie auf den IOD sind. Gängige chirurgische Optionen beim OWG und EWG werden miteinander verglichen, und es wird auf die klinischen Aspekte eingegangen, die jeweils die Wahl des Verfahrens beeinflussen.

\section{Okuläre biometrische Parameter}

\section{Vorhersage des postoperativen IOD}

In den 1970er Jahren berichteten Bigger und Becker über einen niedrigeren IOD nach Kataraktextraktion bei Glaukompatienten [5]. Seitdem hat sich - insbesondere in den letzten 10 Jahren - in zahlreichen Studien durchgängig eine IOD-Senkung unterschiedlichen Ausmaßes nach Kataraktoperationen gezeigt (Tab. 1) [5-18]. Die präoperative Winkelkonfiguration wird als einer der Hauptfaktoren für diese Variabilität angeführt, mit einer höheren IOD-Senkung bei verengtem oder verschlossenem Kammerwinkel. Neben der Winkelanatomie bestand bei zahlreichen weiteren Faktoren ein unabhängiger Zusammenhang mit einem IOD-Rückgang nach Kataraktoperation, darunter die Kammerwasserdynamik, die okuläre Komorbidität sowie insbesondere der präoperative IOD.

Nach der Entfernung der Linse treten selbst in Augen ohne Glaukom anatomische Veränderungen in der Vorderkammer ein, und viele biometrische Faktoren verändern sich. Zum Beispiel sind eine Zunahme der Vorderkammertiefe (VKT), -winkelöffnungs- strecke und -fläche zu erwarten [19-21]. Diese anatomischen Faktoren sind besonders relevant beim EWG, typischerweise durch einen verengten Winkel und eine flache Vorderkammer gekennzeichnet; sie sind umso geringer ausgeprägt, je dicker die Linse wird und je weiter nach anterior sie positioniert ist. Nach einer Phakoemulsifikation zeigen EWG-Patienten einen signifikanten Anstieg der VKT und Winkelbreite im Vergleich zu OWG-Patienten oder auch Kontrollprobanden [21]. Diese biometrischen Veränderungen korrelieren in allen Gruppen mit dem IOD, wobei die IOD-Senkung bei den EWG-Patienten stärker ausgeprägt ist als bei den OWG-Patienten oder der Kontrollgruppe $(p=0,02)$ [10]. Ausgehend von diesen anatomischen und biometrischen Parametern, wurden verschiedene prädiktive Indizes für den postoperativen IOD erarbeitet. Im Jahr 2005 entwickelten Issa et al. [22] einen prädiktiven Index für die IOD-Senkung bei Patienten ohne Glaukom, der auf zwei anatomischen Faktoren basiert: IOD und VKT. Sie gelangten zu dem Schluss, dass dieser Index besser mit der IOD-Veränderung korrelierte als jeder der Parameter für sich genommen. Darüber hinaus legten Liu et al. [23] eine eigene Formel vor, um für Augen mit EWG anhand von IOD und VKT die IOD-Veränderung nach einer Kataraktoperation vorherzusagen $\left(\mathrm{IOD}=6,354+0,186(\mathrm{IOD} \times \mathrm{ACD}) ; \mathrm{R}^{2}=49 \%\right)$. Das Produkt aus IOD und VKT zeigte hohe prädiktive Aussagekraft für den IOD ein Jahr nach der Operation; ein Wert $<35$ entsprach einer höheren Wahrscheinlichkeit, einen IOD $\leq 12 \mathrm{~mm} \mathrm{Hg}$ zu erreichen. Obwohl diese Indizes weder validiert noch global implementiert worden sind, könnten sie bei der Auswahl des chirurgischen Verfahrens zu der Entscheidung beitragen, ob eine einfache Linsenextraktion 
Tab. 2. Konversionsrate von okulärer Hypertension zu Glaukom oder normalem IOD [21]

\begin{tabular}{|c|c|c|c|c|c|c|c|c|}
\hline \multirow[t]{2}{*}{ Studie } & \multirow[t]{2}{*}{$n$} & \multirow{2}{*}{$\begin{array}{l}\text { Nachbeobachtung, } \\
\text { Jahre }\end{array}$} & \multirow[t]{2}{*}{ Behandlung } & \multirow{2}{*}{$\begin{array}{l}\triangle \mathrm{IOP} \\
\mathrm{mm} \mathrm{Hg}\end{array}$} & \multicolumn{2}{|c|}{ Konversion zu Glaukom } & \multicolumn{2}{|c|}{ Konversion zu normalem IOD } \\
\hline & & & & & Faktor & $\%$ & Faktor, mm Hg & $\%$ \\
\hline OHTS [5] & 817 & 5,0 & Medikation & $21-32$ & $\downarrow$ Gesichtsfeld & 4,4 & $1 O D<21$ & 0,0 \\
\hline Poley $[18,27]$ & 81 & 4,1 & Phako + IOL & $20-31$ & Beginn Medikation & 1,1 & $1 \mathrm{IOD}<20$ & 74,7 \\
\hline
\end{tabular}

OHTS = Ocular Hypertensive Treatment Study; Phako = Phakoemulsifikation; IOL = Intraokularlinse. Nach Poley et al. [18].

genügt, um den IOD unter Kontrolle zu bringen, oder ob ein invasiveres (jedoch effektiveres) Vorgehen erforderlich ist. Schließlich sind noch verschiedene weitere biometrische Faktoren als unabhängige Prädiktoren für den postoperativen IOD diskutiert worden - von der Vorderkammerfläche über die Vorderkammerbreite bis zur Linsendicke -, doch ihr Nutzen muss noch in weiterführenden Untersuchungen überprüft werden $[20,24,25]$.

\section{IOD-Senkung bei offenem Vorderkammerwinkel}

Die Abnahme des IOD hängt möglicherweise nicht nur von anatomischen Faktoren verengter Kammerwinkel ab. Die Ergebnisse von Poley et al. [18] deuten darauf hin, dass sich durch die Linsenextraktion die hintere Kapsel nach posterior verlagern und dadurch die Zonula über den Ziliarkörper verschieben kann, was zur Erweiterung des Schlemm-Kanals und zu einer Verbesserung des Kammerwasserabflusses führt. Als weiterer möglicher Mechanismus wird diskutiert, dass die bei der Phakoemulsifikation eingesetzten Ultraschallwellen für einen abrupten Anstieg des Vorderkammerdrucks verantwortlich sind, der mit einer Ausschüttung inflammatorischer Zytokine (hauptsächlich IL-1) einhergeht, die ihrerseits die Produktion von Metalloproteinasen und den Umbau des Trabekelwerks stimulieren und so den Kammerwasserabfluss fördern [26]. Angesichts dieser Ergebnisse sowie der von Poley et al. [27] ist der präoperative IOD der beste Prädiktor für den postoperativen IOD, da die IOD-Veränderung bei OWG-Patienten proportional zur Höhe des präoperativen IOD zu sein scheint [28].

\section{Okuläre Hypertension}

Okuläre Hypertension ist definiert als IOD über Normalwert (21 mm Hg) ohne Gesichtsfeldausfälle oder Sehnervschädigung. Sie ist für sich betrachtet der größte Risikofaktor für die Progression zum Glaukom. Den Ergebnissen der Ocular Hypertensive Treatment Study [5] zufolge bewirkt die Phakoemulsifikation mit Implantation einer Intraokularlinse in dieser Untergruppe von Patienten eine IOD-Senkung proportional zum präoperativen IOD. Poley et al. $[18,27]$ untersuchten die Auswirkungen der Phakoemulsifikation auf die Progression der okulären Hypertension über 10 Jahre und gelangten zu dem Schluss, dass die Kataraktoperation, dank der stärkeren IOD-Senkung, für eine geringere Progressionsrate zum Glaukom verantwortlich sein könnte (Tab. 2). Es mangelt jedoch noch an Evidenz für die Rolle, welche die Kataraktoperation als Präventivmaßnahme für die Progression okulärer Hypertension zum Glaukom spielen könnte.

\section{Engwinkelglaukom}

Primäre Winkelverengung ist definiert als Vorliegen von iridotrabekulärem Kontakt, der den Abfluss des Kammerwassers durch eine diffuse Obstruktion des Trabekelwerks behindert und in der Regel aus einer flachen Vorderkammer und einem gedrängten Winkel des vorderen Segments resultiert. Das Auftreten einer Kammerwinkelverengung ist umso wahrscheinlicher, je geringer der Abstand zwischen Iris und Trabekelwerk ist; die Gefahr des iridotrabekulären Kontakts steigt ab einem Öffnungswinkel von $20^{\circ}$ exponentiell [29]. Aufgrund ihrer intrinsischen Rolle in der Vorderkammeranatomie von Patienten mit primärem Winkelverschluss kann die chirurgische Extraktion der Linse in der kurz- und langfristigen Behandlung des akuten und chronischen EWG (CEWG) von Nutzen sein.

Bei bestehendem akutem Winkelverschluss führt die Linsenextraktion zu einem drastischen Rückgang des IOD (Tab. 1). So wie eine hohe Linsendicke einen Pupillarblock und damit einen Winkelverschluss begünstigt, ermöglicht die Entfernung der Linse die Ausdehnung der Vorderkammer und die Vergrößerung des Winkels, was den Kammerwasserabfluss und die IOD-Senkung fördert. Darüber hinaus bietet diese Behandlungsmethode selbst im Vergleich zur peripheren Laser-Iridotomie (LPI) noch weitere Vorteile (Tab. 3): bessere langfristige IOD-Kontrolle nach Abklingen einer akuten Krise, geringerer Bedarf an Langzeitmedikation zur Aufrechterhaltung eines optimalen IOD, größerer Winkel und weniger periphere anteriore Synechien (PAS) bei geringerem Risiko einer zweiten Winkelverschlusskrise oder einer Progression zu einem chronischen Winkelverschluss, welches nach LPI etwa $58,1 \%$ beträgt $[30,31]$. Es ist jedoch ein riskanteres Vorgehen im Hinblick auf die flachere Vorderkammer, die Empfindlichkeit des Hornhautendothels sowie eine atone Iris [32].

Wenn in einem Auge keine Katarakt vorliegt oder die Sehschärfe gut ist, ist der klinische Nutzen der Kataraktoperation noch umstritten, und die LPI scheint das bessere Risiko-Nutzen-Verhältnis zu haben. Doch wenn eine Katarakt vorliegt, wird die Linsenextraktion als beste Option vorgeschlagen, sobald die akute Krise bewältigt ist [32]. Auch wenn der IOD bei der Vorstellung höher ist als $55 \mathrm{~mm} \mathrm{Hg}$ (ein unabhängiger Faktor für langfristig unkontrollierten IOD), ist mit einer frühen Kataraktextraktion im Vergleich zur LPI die Wahrscheinlichkeit geringer, dass die Patienten später eine IOD-senkende Therapie benötigen [30].

Bei Vorliegen von PAS tritt ein chronischer Verschluss des Vorderkammerwinkels mit permanenter Behinderung des Kammerwas- 
Tab. 3. Vergleich zwischen LPI und Phakoemulsifikation mit Implantation einer Intraokularlinse

\begin{tabular}{lcrrl}
\hline & LPI & Phako & p-Wert & Studie \\
\hline Langzeit-IOD-Kontrolle & & & & \\
$>21$ mm Hg & $46,7 \%$ & $3,3 \%$ & $<0,0001$ & Lam [30], 2008 \\
$<22$ mm Hg & $61,1 \%$ & $89,5 \%$ & 0,034 & Husain [31], 2012 \\
Winkelöffnung (Shaffer) & 0,73 & 2,10 & $<0,0001$ & Lam [30], 2008 \\
Medikationsbedarf für IOD-Kontrolle & 0,90 & 0,03 & $<0,0001$ & Lam [30], 2008 \\
IOD nach 18 Monaten, mm Hg & 15,0 & 12,6 & 0,009 & Lam [30], 2008 \\
PAS, Grad & 228,6 & 101,3 & $<0,0001$ & Lam [30], 2008 \\
\hline
\end{tabular}

Phako $=$ Phakoemulsifikation mit Implantation einer Intraokularlinse. serabflusses ein. Der IOD hängt nun nahezu vollständig vom Ausmaß der Schädigung des Trabekelwerks ab, welche entweder unmittelbar durch die PAS hervorgerufen wird oder als indirekte Folge des erhöhten IOD (ob durch hypertensive Spikes oder kontinuierliche Hypertension) auftritt. Der IOD bleibt kontinuierlich erhöht, wenn mehr als $180^{\circ}$ des Winkels durch PAS okkludiert sind; wenn der Wert $270^{\circ}$ übersteigt, ist eine medikamentöse Therapie in der Regel wirkungslos, und eine Filtrationschirurgie wird erforderlich [33].

Die traditionelle Vorgehensweise bei Patienten mit primärem EWG ist eine gestaffelte Kombination von medikamentöser und Lasertherapie; wenn diese versagen, ist als Zweitlinientherapie eine Glaukomoperation angezeigt. Die Kataraktextraktion wird jedoch zunehmend auch zur primären EWG-Behandlung in Betracht gezogen, da sie die Anatomie der Vorderkammer durch die Linsenextraktion wiederherstellen kann und der Patient häufig ohne weitere Medikamente auskommt.

Bei primären EWG-Patienten mit ungetrübter Linse sind sowohl Phakoemulsifikation als auch Trabekulektomie wirksame Mittel zur Senkung des IOD (Reduktion um 8,4 mm Hg bzw. 34\% durch Phakoemulsifikation vs. $8,9 \mathrm{~mm} \mathrm{Hg}$ bzw. $36 \%$ durch Trabekulektomie; $p>0,05)$. Die mit Trabekulektomie behandelten Patienten schienen einen geringeren Bedarf an zusätzlicher Medikation zu haben als die mit Phakoemulsifikation behandelten Patienten (wenn auch nicht in statisch signifikantem Maße; $\mathrm{p}=0,16$ ); $\mathrm{zu}-$ gleich war die Trabekulektomie mit signifikant mehr chirurgischen Komplikationen assoziiert als die Phakoemulsifikation (46 vs. $4 \% ; p=0,001$ ) [34]. Die häufigste langfristige Komplikation ist die Kataraktbildung (33\%), die einen weiteren chirurgischen Eingriff zur Wiederherstellung der Sehschärfe erforderlich macht [34]. Schätzungen zufolge wird außerdem bei der Hälfte aller Patienten mit CEWG, die sich einer Trabekulektomie unterziehen, innerhalb von 5 Jahren eine Linsenextraktion erforderlich [35].

Auf der Grundlage dieser klinischen Studien ist diskutiert worden, ob eine frühe Extraktion der ungetrübten Linse von klinischem Nutzen für die Kontrolle von mittelschwerem CEWG oder sogar für die CEWG-Prävention wäre. In einer Meta-Analyse von 2006 gelangen Friedman und Vedula [36] zu dem Schluss, dass es keine belastbaren Belege gibt, die für eine solche klare Linsenextraktion zur Glaukombehandlung sprechen. Neuere klinische Studien sind inzwischen jedoch zu einer anderen Auffassung gelangt und befürworten die Phakoemulsifikation als wertvolle Option, die sicherer, schneller und wirtschaftlicher ist als jede andere chirurgische Maßnahme zur Behandlung des CEWG. Zur CEWG-Prophylaxe bei
Patienten mit vermutetem oder chronischem Winkelverschluss hingegen spielt die klare Linsenextraktion derzeit keine Rolle [37]. Um diese Frage zu klären, wird derzeit die EAGLE-Studie durchgeführt (Effectiveness of Early Lens Extraction with Intraocular Lens Implantation for the Treatment of Primary Angle-Closure Glaucoma). In der 2011 begonnenen, multizentrischen, prospektiven, randomisierten klinischen Studie werden Extraktion der klaren Linse und LPI bei Patienten mit neu diagnostiziertem chronischem Winkelverschluss oder CEWG verglichen [38]. Die primären Endpunkte sind IOD, Lebensqualität des Patienten und Kosteneffizienz; der Nachbeobachtungszeitraum umfasst 3 Jahre. Danach wird es eventuell möglich sein, den Nutzen der Phakoemulsifikation in der gängigen Praxis der CEWG-Behandlung zu bestätigen.

Neben Phakoemulsifikation und Trabekulektomie gibt es eine dritte chirurgische Option, die beide Verfahren miteinander verbindet und als Phakotrabekulektomie oder kombinierte Operation bezeichnet wird. Im Vergleich zur Phakoemulsifikation scheint die kombinierte Operation geringfügig wirksamer in Bezug auf den IOD $(p>0,05)$ zu sein, bei geringerem Medikationsbedarf. Zugleich ist die Rate der chirurgischen Komplikationen in der mit der kombinierten Chirurgie behandelten Gruppe signifikant höher, ebenso die Progressionsrate der optischen Neuropathie $[39,40]$. Der kleine Benefit des geringeren Medikationsbedarfs nach der Trabekulektomie und der stärkeren IOD-Senkung nach der kombinierten Operation wird durch die höhere Rate chirurgischer Komplikationen zunichte gemacht. Lediglich in Situationen, in denen minimale Medikation eine Priorität darstellt, ist die Trabekulektomie weiterhin die adäquatere Option [41]. Mehrere Autoren sind der Auffassung, dass bei fortgeschrittenem Glaukom die kombinierte Operation die beste Option darstellt, da sie eine bessere IOD-Kontrolle bewirkt als die Phakoemulsifikation und im Vergleich zur Trabekulektomie ähnlich wirksam den IOD kontrolliert und den Bedarf an Glaukommedikamenten reduziert, bei geringerem Bedarf an erneuten Eingriffen [42].

\section{Offenwinkelglaukom}

Der IOD-Anstieg beim OWG hängt nicht mit einer Verengung des Kammerwinkels zusammen, sondern mit einer Störung des Kammerwasserabflusses im Trabekelwerk. Eine medikamentöse Behandlung ist in der Mehrzahl der Fälle die erste Therapielinie; eine Filtrationschirurgie kommt ins Spiel, wenn der Ziel-IOD nicht erreicht oder, im Falle von jungen Patienten oder schlechter 
Verträglichkeit der medikamentösen Behandlung, voraussichtlich nicht dauerhaft aufrechterhalten werden kann. Die Filtrationschirurgie ist allerdings wahrscheinlich nicht die einzige Operation, der ein Glaukompatient sich unterziehen muss. Bei 30\% aller Medicare-Patienten in den USA, bei denen eine Kataraktoperation vorgenommen wurde, lag gleichzeitig auch ein Glaukom vor [43] Dieser bedeutsame epidemiologische Faktor macht es umso wichtiger, diese Operation in die Praxisalgorithmen des Glaukommanagements zu integrieren. Ähnlich wie beim CEWG stehen drei chirurgische Optionen zur Wahl: Phakoemulsifikation, Trabekulektomie und die kombinierte Operation (Phakotrabekulektomie). Je nach Schweregrad des Glaukoms ist das therapeutische Vorgehen individuell auf den Patienten zuzuschneiden:

Leichtes Glaukom. Bei Patienten, die unter Kataraktbeschwerden leiden, aber ein geringgradiges, gut kontrolliertes Glaukom mit geringem Medikationsbedarf und geringer Sehnervschädigung haben, ist die alleinige Phakoemulsifikation eine zuverlässige Option. Die Linsenentfernung verbessert die Sehschärfe und kann die Beurteilung und Nachbeobachtung des Krankheitsgeschehens erleichtern. Darüber ist eine geringfügige IOD-senkende Wirkung der Phakoemulsifikation möglich, die zur IOD-Kontrolle beiträgt - im Durchschnitt beträgt die Reduktion 1,5-3,0 mm Hg (Tab. 1). Jedoch ist weiterhin ein Risiko für Komplikationen, insbesondere hypertensive Spikes, mit dieser Operation verbunden.

Mittelschweres Glaukom. Bei dieser Patientenpopulation richtet sich die klinische Entscheidungsfindung besonders stark nach individuellen Merkmalen. Die Phakoemulsifikation ist auch hier eine wertvolle chirurgische Option bei Patienten mit ansonsten guter IOD-Kontrolle, wobei hier besondere Aufmerksamkeit wegen der häufigeren hypertensiven Spikes erforderlich ist. Bei einem IOD über dem Zielwert sind die Vor- und Nachteile einer kombinierten Operation mit dem Patienten zu besprechen. Einerseits könnten durch die Kombination aus Kataraktoperation und Trabekulektomie zwei Probleme mit einem einzigen Eingriff angegangen werden (da eine Katarakt- nach einer Filtrationsoperation fast unvermeidlich ist), und die hypertensiven Spikes nach einer Phakoemulsifikation könnten vermieden werden. Andererseits ist die kombinierte Chirurgie mit mehr postoperativen Komplikationen und einer weniger bedeutsamen IOD-Senkung assoziiert als die alleinige Trabekulektomie $[44,45]$. Hierbei ist allerdings anzumerken, dass Patienten mit moderatem Glaukom tendenziell einen höheren IOD-Zielwert haben, was sie zu guten Kandidaten für eine einmalige Kombinationsoperation machen würde. Bei beginnender Entstehung einer Katarakt und mittelschwerem Glaukom oder wenn der IOD nicht im optimalen Bereich liegt, ist das vorrangige Ziel, das Glaukom unter Kontrolle zu bringen. Die chirurgische Erstlinientherapie ist die Trabekulektomie, doch auch andere, weniger kataraktogene Optionen können in Betracht gezogen werden, insbesondere nichtpenetrierende Verfahren wie die tiefe Sklerektomie und Viskokanalostomie, trotz ihrer im Vergleich zur Trabekulektomie geringeren Erfolgsrate [46].

Fortgeschrittenes Glaukom. Bei schwerem Glaukom besteht ein hohes Risiko für Sehverluste infolge von IOD-Spikes oder -Schwankungen; hier hat eine aggressivere IOD-senkende Strategie Priori- tät. Die beste chirurgische Option ist eine Trabekulektomie mit nachfolgender Kataraktentfernung - der sequenzielle Ansatz. Die Linsenextraktion sollte in einem zweiten chirurgischen Eingriff mindestens 6 Monate und idealerweise 2 Jahre später erfolgen, wobei diese Verzögerung mit dem Preis einer deutlichen Verringerung der Filtrationseffizienz nach der Trabekulektomie verbunden ist [47]. Hierbei ist zu bedenken, dass in Sonderfällen, wenn Patienten z.B. aufgrund ihres Alters, wegen Begleiterkrankungen oder operativer/anästhetischer Gegenanzeigen nicht für zwei separate OP-Termine in Frage kommen, auch die kombinierte Operation in Betracht gezogen werden kann. In ähnlicher Weise argumentieren andere Autoren, dass angesichts der Gesamtwirkung der Phakoemulsifikation auf ein früheres Sickerkissen das Ergebnis der beiden chirurgischen Vorgehensweisen (kombiniert vs. sequenziell) sehr ähnlich sein dürfte. Die einzige klinische Studie, welche die beiden chirurgischen Vorgehensweisen vergleicht, ergibt ähnliche IOD-Werte. Allerdings werden hier die präoperativen IOD-Werte nicht genannt, daher ist keine Aussage über das Ausmaß der IODSenkung durch die beiden Vorgehensweisen möglich [48].

\section{Neuartige chirurgische Ansätze}

Eine Reihe neuer chirurgischen Alternativen hat sich in den letzten Jahren entwickelt. Die neuen Verfahren sind minimalinvasiv und meist darauf ausgelegt, in Kombination mit einer Kataraktoperation angewandt zu werden, um eine zusätzliche Senkung des IOD und des Bedarfs an Glaukommedikation zu bewirken. $\mathrm{Zu}$ diesen Verfahren zählen die endoskopische Zyklophotokoagulation, der trabekuläre Mikro-Bypass-Stent (iStent), die Trabekulotomie ab interno (Trabectome), die Kanaloplastik sowie zwei jüngere Ab-interno-Methoden (Hydrus und CyPass).

Die endoskopische Zyklophotokoagulation umfasst die Laserkoagulation der Ciliarfortsätze und ermöglicht in Kombination mit der Phakoemulsifikation eine IOD-Senkung um 17,6-46,9\% [49]. Der Großteil der Daten hierzu stammt allerdings aus retrospektiven, unkontrollierten Fallberichten; nur eine kontrollierte randomisierte Studie berichtet von einem signifikanten Rückgang der IOD-Werte und Glaukommedikation in der Behandlungsgruppe (17,6 vs. $4,2 \%$ IOD-Senkung und 57,5 vs. $0 \%$ weniger Medikation) über einen Nachbeobachtungszeitraum von 3 Jahren [50]. Bedenken bereitet außerdem die hohe Rate postoperativer Komplikationen im Vergleich zu Drainagesystemen.

Der iStent (Glaukos Corp., Laguna Hills, CA, USA) ist ein Medizinprodukt, das intern in den Schlemm-Kanal eingeführt wird und das Kammerwasser unter Umgehung des Trabekelwerks direkt aus der Vorderkammer in den Schlemm-Kanal ableitet. Die größte kontrollierte, randomisierte klinische Studie hierzu berichtete von einem hohen Anteil an Patienten, die nach 2 Jahren Nachbeobachtung einen IOD $<21 \mathrm{~mm} \mathrm{Hg}$ erreichten (61 vs. 50\%; $\mathrm{p}=0,036$ ) [51]. Zusätzlich wurde bei $81,3 \%$ ein geringerer Verbrauch an IOD-senkenden Medikamenten festgestellt.

Bei der Ab-interno-Trabekulotomie wird mit dem Trabektom (NeoMedix Inc., Tustin, CA, USA) mittels Elektrokauterisation ein Teil des Trabekelwerks entfernt. Mehrere retrospektive, un- 
kontrollierte Studien berichten von einer IOD-Senkung um 4-7 mm Hg durch die kombinierte Anwendung des Trabektoms und der Phakoemulsifikation sowie von einem erheblichen Rückgang des Bedarfs an Glaukommedikation [49]. Zur IOD-senkenden Wirkung des Trabektom-Verfahrens allein liegt jedoch noch keine beweiskräftige Evidenz vor.

Die Kanaloplastik (iScience Interventional Corp., Menlo Park, CA, USA) umfasst die Kanülierung des Schlemm-Kanals mit nachfolgender Dehnung des Trabekelwerks; dies scheint ein neuartiger Ansatz mit einer höheren IOD-Senkung (40\% bzw. $10 \mathrm{~mm}$ $\mathrm{Hg}$ ) zu sein, wenn man ihn in Kombination mit der Phakoemulsifikation anwendet; assoziierte Nachteile sind jedoch Bindehautvernarbungen, welche die Wirksamkeit einer nachfolgenden Trabekulektomie beschränken könnten, sowie eine höhere Rate chirurgischer Komplikationen [52].

Hydrus (Ivantis Inc., Irvine, CA, USA) ist ein neuartiges Gerüst für den Schlemm-Kanal, das den Kammerwasserabfluss verbessert. Eine vorläufige Auswertung einer 6-monatigen Nachbeobachtungsstudie lieferte vielversprechende Ergebnisse (durchschnittlich 4,7 mm Hg IOD-Senkung) dank des doppelten Wirkungsmechanismus: Perforation des Trabekelwerks und Erweiterung des Schlemm-Kanals [53]. CyPass (Transcend Medical, Menlo Park, CA, USA) ist ein Polyamid-Implantat, das durch die Kammerwinkelregion in den Suprachoroidalraum eingebracht wird, um den uveoskleralen Abfluss zu fördern. Die veröffentlichten Ergebnisse belegen eine IOD-Senkung um 37\% in nicht medikamentös eingestellten Augen und einen um 50\% geringeren Bedarf an Glaukommedikation nach 6 Monaten Nachbeobachtung bei sehr geringen Nebenwirkungsraten [54]. Diese neuartigen Ansätze eignen sich am besten für die Anwendung in Kombination mit Phakoemulsifikation bei gering- bis mittelgradigem primärem OWG (POWG), wenn ein geringeres $\mathrm{Maß}$ an IOD-Senkung ausreicht. Belastbare randomisierte, kontrollierte klinische Studien, die diese kombinierten Ansätze mit der alleinigen Phakoemulsifikation vergleichen, stehen jedoch für die Mehrheit der neuen Ansätze noch aus, und ihr wahres Potenzial für die Patientenversorgung ist bisher nicht klar zu benennen.

\section{Schlussfolgerung}

Es liegen Hinweise dafür vor, dass die Kataraktoperation zu einer mäßig lang anhaltenden IOD-Senkung sowohl bei POWG- als auch bei CEWG-Patienten führt. Aufgrund der inhärenten anatomischen Merkmale der verschiedenen Arten von Glaukomen ist dieser hypotensive Effekt ausgeprägter in Augen mit CEWG, bei denen die Linsenextraktion zur Aufweitung der Vorderkammer führt. Die Phakoemulsifikation spielt eine zunehmend wichtige Rolle in der CEWG-Kontrolle, da sie den anatomischen Umbau der Vorderkammer bewirken kann, und es wird derzeit diskutiert, ob die Extraktion der ungetrübten Linse ein günstiges Risiko-Nutzen-Verhältnis für die CEWGPrävention und/oder -Behandlung aufweist.

Im Hinblick auf die okuläre Hypertension bei POWG hingegen, die primär auf Funktionsstörungen des Trabekelwerks zurückzuführen ist, bewirkt eine Kataraktoperation ein geringeres Maß an IOD-Senkung; der hier zugrunde liegende Mechanismus ist bisher im Wesentlichen ungeklärt. Bei diesen Patienten wirkt sich der Eingriff nur geringfügig auf die IOD-Kontrolle aus, jedoch kann auch diese bescheidene und variable IOD-Senkung noch klinisch bedeutsam sein und dem Patienten helfen, seinen ZielIOD zu erreichen. Bei der Versorgung von Patienten mit fortgeschrittenem Glaukom und unkontrolliertem IOD hingegen sollte eine aggressivere IOD-senkende Strategie Vorrang haben - z.B. in Form einer Filtrationsoperation -, und die Kataraktoperation sollte zunächst aufgeschoben werden. Die kombinierte Operation oder Phakotrabekulektomie ist ein chirurgischer Eingriff, der bisher ohne formelle Indikation ist, jedoch in bestimmten Situationen sinnvoll sein könnte. Gut aufgebaute, große, prospektive, randomisierte Studien hierzu stehen noch aus, doch die bisher verfügbaren Daten legen nahe, dass dieser gängige Eingriff seinen Platz in der Glaukombehandlung finden wird.

\section{Disclosure Statement}

Bei keinem der Autoren besteht ein Interessenkonflikt im Hinblick auf die Einreichung, und es gab keine finanzielle Unterstützung.

\section{Literatur}

1 Pascolini D, Mariotti SP: Global estimates of visual impairment: 2010. Br J Ophthalmol 2012;96:614-618.

2 Quigley HA, Broman AT: The number of people with glaucoma worldwide in 2010 and 2020. Br J Ophthalmol 2006;90:262-267.

3 Asbell PA, Dualan I, Mindel J, Brocks D, Ahmad M, Epstein S: Age-related cataract. Lancet 2005;365:599-609.

4 Shrivastava A, Singh K: The effect of cataract extraction on intraocular pressure. Curr Opin Ophthalmol 2010;21:118-122.

5 Mansberger SL, Gordon MO, Jampel HD, Bhorade A, Brandt JD, Wilson B, Kass MA: Reduction in intraocular pressure after cataract extraction: the Ocular Hypertension Treatment Study. Ophthalmology 2012;119:1826-1831.
6 Pohjalainen T, Vesti E, Uusitalo RJ, Laatikainen L: Intraocular pressure after phacoemulsification and intraocular lens implantation in nonglaucomatous eyes with and without exfoliation. J Cataract Refract Surg 2001;27:426-431.

7 Shingleton BJ, Pasternack JJ, Hung JW, O'Donoghue MW: Three and five year changes in intraocular pressures after clear corneal phacoemulsification in open angle glaucoma patients, glaucoma suspects, and normal patients. J Glaucoma 2006;15:494-498.

8 Jacobi PC, Dietlein TS, Lüke C, Engels B, Krieglstein GK: Primary phacoemulsification and intraocular lens implantation for acute angle-closure glaucoma. Ophthalmology 2002; 109:1597-1603.
9 Su W-W, Chen PY-F, Hsiao C-H, Chen HS-L: Primary phacoemulsification and intraocular lens implantation for acute primary angle-closure. PLoS One 2011;6:e20056.

10 Hayashi K, Hayashi H, Nakao F, Hayashi F: Effect of cataract surgery on intraocular pressure control in glaucoma patients. J Cataract Refract Surg 2001;27:1779-1786.

11 Lai JS, Tham CC, Chan JC, Lam DS: Phacotrabeculectomy in treatment of primary angleclosure glaucoma and primary open-angle glaucoma. Jpn J Ophthalmol 2004;48:408-411.

12 Liu CJ, Cheng CY, Wu C-W, Lau L-I, Chou JC, Hsu W-M: Factors predicting intraocular pressure control after phacoemulsification in angle-closure glaucoma. Arch Ophthalmol 2006; 124:1390-1394. 
13 Lai JS, Tham CC, Chan JC: The clinical outcomes of cataract extraction by phacoemulsification in eyes with primary angle-closure glaucoma (PACG) and co-existing cataract: a prospective case series. J Glaucoma 2006;15:47-52.

14 Kim DD, Doyle JW, Smith MF: Intraocular pressure reduction following phacoemulsification cataract extraction with posterior chamber lens implantation in glaucoma patients. Ophthalmic Surg Lasers 1999;30:37-40.

15 Shingleton B, Gamell L, O’Donoghue MW, Baylus SL, King R: Long-term changes in intraocular pressure after clear corneal phacoemulsification: normal patients versus glaucoma suspect and glaucoma patients. J Cataract Refract Surg 1999;25:885-890.

16 Pohjalainen T, Vesti E, Uusitalo RJ, Laatikainen L: Phacoemulsification and intraocular lens implantation in eyes with open-angle glaucoma. Acta Ophthalmol Scand 2001;79:313-316.

17 Mathalone N, Hyams M, Neiman S, Buckman G, Hod Y, Geyer O: Long-term intraocular pressure control after clear corneal phacoemulsification in glaucoma patients. J Cataract Refract Surg 2005;31:479-483.

18 Poley BJ, Lindstrom RL, Samuelson TW, Schulze $\mathrm{R}$ : Intraocular pressure reduction after phacoemulsification with intraocular lens implantation in glaucomatous and nonglaucomatous eyes: evaluation of a causal relationship between the natural lens and open-angle glaucoma. J Cataract Refract Surg 2009;35:1946-1955.

19 Yang HS, Lee J, Choi S: Ocular biometric parameters associated with intraocular pressure reduction after cataract surgery in normal eyes. Am J Ophthalmol 2013;156:89-94.

20 Huang G, Gonzalez E, Lee R, Chen Y-C, He M, Lin SC: Association of biometric factors with anterior chamber angle widening and intraocular pressure reduction after uneventful phacoemulsification for cataract. J Cataract Refract Surg 2012;38:108-116.

21 Hayashi K, Hayashi H, Nakao F, Hayashi F: Changes in anterior chamber angle width and depth after intraocular lens implantation in eyes with glaucoma. Ophthalmology 2000;107: 698-703.

22 Issa S, Pacheco J, Mahmood U, Nolan J, Beatty S: A novel index for predicting intraocular pressure reduction following cataract surgery. Br J Ophthalmol 2005;89:543-546.

23 Liu CJ, Cheng C-Y, Ko Y-C, Lau L-I: Determinants of long-term intraocular pressure after phacoemulsification in primary angle-closure glaucoma. J Glaucoma 2011;20:566-570.

24 Pradhan S, Leffler CT, Wilkes M, Mahmood MA: Preoperative iris configuration and intraocular pressure after cataract surgery. J Cataract Refract Surg 2012;38:117-123.

25 Shrivastava A, Singh K: The impact of cataract surgery on glaucoma care. Curr Opin Ophthalmol 2014;25:19-25.

26 Wang N, Chintala SK, Fini ME, Schuman JS: Ultrasound activates the TM ELAM-1/IL-1/ NF- $\kappa B$ response: a potential mechanism for intraocular pressure reduction after phacoemulsification. Invest Ophthalmol Vis Sci 2003;44 1977-1981.
27 Poley BJ, Lindstrom RL, Samuelson TW: Longterm effects of phacoemulsification with intraocular lens implantation in normotensive and ocular hypertensive eyes. J Cataract Refract Surg 2008;34:735-742.

28 Guan H, Mick A, Porco T, Dolan BJ: Preoperative factors associated with IOP reduction after cataract surgery. Optom Vis Sci 2013;90: 179-184.

29 Foster PJ, Aung T, Nolan WP, Machin D, Baasanhu J, Khaw PT, Alsbirk P-H, Lee PS, Seah SKL, Johnson GJ: Defining 'occludable' angles in population surveys: drainage angle width, peripheral anterior synechiae, and glaucomatous optic neuropathy in east Asian people. $\mathrm{Br}$ J Ophthalmol 2004;88:486-490.

30 Lam DS, Leung DY, Tham CC, Li FC, Kwong YY, Chiu TY, Fan DS: Randomized trial of early phacoemulsification versus peripheral iridotomy to prevent intraocular pressure rise after acute primary angle closure. Ophthalmology 2008;115:1134-1140.

31 Husain R, Gazzard G, Aung T, Chen Y, Padmanabhan V, Oen FTS, Seah SKL, Hoh S-T: Initial management of acute primary angle closure: a randomized trial comparing phacoemulsification with laser peripheral iridotomy. Ophthalmology 2012;119:2274-2281.

32 Lachkar Y: Acute angle closure and angle closure glaucoma: phacoemulsification as firstline treatment (in French). J Fr Ophtalmol 2010;33:273-278.

33 Teekhasaenee C, Ritch R: Combined phacoemulsification and goniosynechialysis for uncontrolled chronic angle-closure glaucoma after acute angle-closure glaucoma. Ophthalmology 1999;106:669-674.

34 Tham CC, Kwong YY, Baig N, Leung DY, Li FC, Lam DS: Phacoemulsification versus trabeculectomy in medically uncontrolled chronic angle-closure glaucoma without cataract. Ophthalmology 2013;120:62-67.

35 AGIS Investigators: The Advanced Glaucoma Intervention Study: 8. Risk of cataract formation after trabeculectomy. Arch Ophthalmol 2001;119:1771-1779.

36 Friedman DS, Vedula SS: Lens extraction for chronic angle-closure glaucoma. Cochrane Database Syst Rev 2006;3:CD005555.

37 Thomas R, Walland MJ, Parikh RS: Clear lens extraction in angle closure glaucoma. Curr Opin Ophthalmol 2011;22:110-114.

38 Azuara-Blanco A, Burr JM, Cochran C, Ramsay C, Vale L, Foster P, Friedman D, Quayyum Z, Lai JS, Nolan W, Aung T, Chew P, McPherson G, McDonald A, Norrie J; Effectiveness in Angleclosure Glaucoma of Lens Extraction (EAGLE) Study Group: The effectiveness of early lens extraction with intraocular lens implantation for the treatment of primary angle-closure glaucoma (EAGLE): study protocol for a randomized controlled trial. Trials 2011;12:133.

39 Tham CC, Kwong YY, Leung DY, Lam SW, Li FC, Chiu TY, Chan JC, Chan CH, Poon AS, Yick DW, Chi CC, Lam DS, Lai JS: Phacoemulsification versus combined phacotrabeculectomy in medically controlled chronic angle closure glaucoma with cataract. Ophthalmology 2008;115:2167-2173.
40 Tham CC, Kwong YY, Leung DY, Lam SW, Li FC, Chiu TY, Chan JC, Lam DS, Lai JS: Phacoemulsification versus combined phacotrabeculectomy in medically uncontrolled chronic angle closure glaucoma with cataracts. Ophthalmology 2009;116:725-731.

41 American Academy of Ophthalmology Glaucoma Panel: Preferred Practice Pattern ${ }^{\circledR}$ Guidelines. Primary Angle Closure. San Francisco, 2010.

42 Tsai H-Y, Liu CJ, Cheng C-Y: Combined trabeculectomy and cataract extraction versus trabeculectomy alone in primary angle-closure glaucoma. Br J Ophthalmol 2009;93:943-948.

43 Cullen KA, Hall MJ, Golosinskiy A: Ambulatory Surgery in the United States, 2006. Hyattsville, 2009.

44 Lochhead J, Casson RJ, Salmon JF: Long term effect on intraocular pressure of phacotrabeculectomy compared to trabeculectomy. $\mathrm{Br} \mathrm{J}$ Ophthalmol 2003;87:850-852.

45 Ogata-Iwao M, Inatani M, Takihara Y, Inoue T, Iwao $\mathrm{K}$, Tanihara $\mathrm{H}$ : A prospective comparison between trabeculectomy with mitomycin $\mathrm{C}$ and phacotrabeculectomy with mitomycin C. Acta Ophthalmol 2013;91:e500-e501.

46 European Glaucoma Society: Terminology and Guidelines for Glaucoma, ed 3. Savona, Dogma, 2008

47 Husain R, Liang S, Foster PJ, Gazzard G, Bunce C, Chew PTK, Oen FTS, Khaw PT, Seah SKL, Aung T: Cataract surgery after trabeculectomy: the effect on trabeculectomy function. Arch Ophthalmol 2012;130:165-170.

48 Donoso R, Rodríguez A: Combined versus sequential phacotrabeculectomy with intraoperative 5-fluorouracil. J Cataract Refract Surg 2000;26:71-74.

49 Budenz DL, Gedde SJ: New options for combined cataract and glaucoma surgery. Curr Opin Ophthalmol 2014;25:141-147.

50 Berke SJ: Endolaser cyclophotocoagulation in glaucoma management. Tech Ophthalmol 2006;4:74-81

51 Craven ER, Katz LJ, Wells JM, Giamporcaro JE: Cataract surgery with trabecular micro-bypass stent implantation in patients with mildto-moderate open-angle glaucoma and cataract: two-year follow-up. J Cataract Refract Surg 2012;38:1339-1345.

52 Augustinus CJ, Zeyen T: The effect of phacoemulsification and combined phaco/glaucoma procedures on the intraocular pressure in open-angle glaucoma. A review of the literature. Bull Soc Belge Ophtalmol 2012;51-66.

53 Radcliffe NM, Lynch MG, Brown RH: Ab interno stenting procedures. J Cataract Refract Surg 2014;40:1273-1280.

54 Hoeh H, Ahmed IIK, Grisanti S, Grisanti S, Grabner G, Nguyen QH, Rau M, Yoo S, Ianchulev T: Early postoperative safety and surgical outcomes after implantation of a suprachoroidal micro-stent for the treatment of openangle glaucoma concomitant with cataract surgery. J Cataract Refract Surg 2013;39: 431-437. 